\title{
Anthraquinone-mediated reduction of a trithiocarbonate chain transfer agent to initiate electrochemical Reversible Addition- Fragmentation Chain Transfer (eRAFT) polymerization
}

Lisa T. Strover, ${ }^{1, *}$ Almar Postma, ${ }^{1}$ Michael D. Horne,${ }^{2}$ Graeme Moad ${ }^{1, *}$

\author{
${ }^{1}$ CSIRO Manufacturing, Clayton, VIC 3168, Australia \\ ${ }^{2}$ CSIRO Mineral Resources, Clayton, VIC 3168, Australia \\ *Corresponding authors: lisa.strover@csiro.au; graeme.moad@csiro.au
}




\section{EXPERIMENTAL SECTION}

\section{Materials}

RAFT agents 4-cyano-4-(((dodecylthio)carbonothioyl)thio)pentanoic acid (CDTPA), 2-cyanobutan-2-yl 3,5dimethyl-1H-pyrazole-1-carbodithioate, and dibenzyl trithiocarbonate were purchased from Boron Molecular; 2cyano-2-propyl dodecyl trithiocarbonate was purchased from STREM; cyanomethyl benzodithioate was purchased from Aldrich; and cyanomethyl dodecyl trithiocarbonate ${ }^{23}$ was kindly provided by former researchers at CSIRO. The structure and purity of RAFT agents was confirmed by ${ }^{1} \mathrm{H}$ and ${ }^{13} \mathrm{C}$ NMR. ${ }^{12}$ Tetrabutylammonium hexafluorophosphate $\left(\mathrm{TBAPF}_{6}\right)$, tetraethylammonium tetrafluoroborate $\left(\mathrm{TEABF}_{4}\right)$, silver nitrate $\left(\mathrm{AgNO}_{3} ; \mathrm{BASi}\right)$, s-trioxane (Acros Organics 99+ \%), and anthraquinone (AQ; BDH Laboratory Reagent) were obtained from commercial suppliers and used without further purification. Solvents (anhydrous or HPLC grade) were used as received. Monomers methyl methacrylate (MMA; Aldrich $99 \%$ inhibited with $\leq 30$ ppm MEHQ), $n$-butyl acrylate (BA; $\geq 99 \%$, inhibited with 10-60 ppm MEHQ), styrene (St; ReagentPlus, $\geq 99 \%$, with 4-tert-butyl catchetol as stabilizer) and $N, N$-dimethylacrylamide (DMA; $99 \%$, inhibited with 500 ppm MEHQ), purchased from Aldrich, were filtered through basic alumina; and $N$ vinyl pyrrolidone (NVP; $\geq 99 \%$, with sodium hydroxide as inhibitor) was filtered through acidic alumina to remove inhibitor prior to polymerization.

\section{Cyclic voltammetry (CV)}

CVs of RAFT agents and AQ were conducted with an Autolab PGSTAT 302N potentiostat, in a nitrogen-purged 3electrode cell with a glassy carbon (GC) rod counter electrode (C.E.), $1 \mathrm{mM}$ GC disk working electrode (W.E.), and Ag wire pseudo-reference electrode (pseudoR.E.). The working electrode was polished with $0.5 \mu \mathrm{M}$ alumina slurry and thoroughly rinsed before each CV experiment. CVs were conducted in ACN with $6 \mathrm{mM} \mathrm{RAFT}$ agent, $0.6 \mathrm{mM} \mathrm{AQ}$, and 0.1 $\mathrm{M} \mathrm{TBAPF}_{6}$ as supporting electrolyte.

\section{General procedure for eRAFT polymerization}

eRAFT polymerizations and control experiments (without mediator or RAFT agent, or at open circuit potential (OCP)) were conducted in a 5-necked flask in a 3-electrode cell configuration, with a GC rod C.E. separated from the bulk solution by a fritted glass tube, GC rod W.E., and an $\mathrm{Ag} / \mathrm{AgNO}_{3}(0.01 \mathrm{M})$ reference electrode (R.E.) (Figure 2). Experiments were controlled and monitored with an Autolab PGSTAT 302N potentiostat. All experiments were conducted by potentiostatic electrolysis with $0.1 \mathrm{M} \mathrm{TEABF}_{4}$ as supporting electrolyte, and $50 \mathrm{mM}$ trioxane as NMR standard. In a typical experiment (target DP = 100), monomer (MMA, $5.0 \mathrm{M}, 100$ equiv.), mediator $(\mathrm{AQ}, 5 \mathrm{mM}, 0.1$ equiv.), and RAFT agent (CDTPA, $50 \mathrm{mM}, 1$ equiv.) were added to the electrochemical cell with $\mathrm{TEABF}_{4}$, trioxane, 27.9 mL DMSO, and a stir bar. The cell was sealed with rubber septa and purged with nitrogen for approx. 30 min. Prior to electrolysis, a CV was recorded to determine the peak reduction potential $\left(E_{\mathrm{pc}}\right)$ of AQ. Polymerization was performed at a constant applied potential of $E_{\mathrm{pc} A \mathrm{AQ}}$ for $24 \mathrm{hr}$ in the unheated cell, under a constant low flow of nitrogen above the solution, with stirring at 500 RPM. Aliquots were taken periodically to monitor conversion and the product was precipitated in $\mathrm{MeOH}$ /water. The same general procedure was used for all experiments, with variations in conditions as described in Table 1. All experiments were conducted with DMSO as solvent and at ambient temperature, unless otherwise stated in Table 1 footnotes.

\section{Chain extension and block copolymer synthesis from eRAFT-synthesized macroCTA}

A RAFT agent-terminated macroCTA (TC-PMMA; $M_{\mathrm{n}}=8.7 \times 10^{-3}$ PMMA equiv., $\theta=1.15$ ) was synthesized by eRAFT as described above, in DMSO with a target DP of 100. Following precipitation and drying of the macroCTA, chain extension with MMA was performed by eRAFT (MMA:AQ:macroCTA = 100:1:0.1) under ambient temperature for $3 \mathrm{hr}$. The macroCTA was also used as the PMMA block in poly(methyl methacrylate)- $b$-polystyrene (PMMA- $b$-PSt), with the PSt block polymerized by conventionally initiated RAFT polymerization as follows. Styrene ( $5.5 \mathrm{~mL} 400$ equiv.), TCPMMA (1.04 g, 1 equiv.), and AIBN ( $4.6 \mathrm{mg}, 0.2$ equiv.), and trioxane (86.5 mg) were dissolved in dioxane ( $4.5 \mathrm{~mL})$. The solution was purged with nitrogen for $30 \mathrm{~min}$. and polymerization was then carried out at $90{ }^{\circ} \mathrm{C}$ for $90 \mathrm{~min}$. The product was collected by precipitation in $\mathrm{MeOH} /$ water.

\section{Polymer characterization}

All NMR spectra were recorded with a Bruker AV 400 or $500 \mathrm{MHz}$ spectrometer at room temperature with $\mathrm{CDCl}_{3}$ as solvent. Gel permeation chromatography (GPC) was performed on a Shimadzu system equipped with a CMB-20A controller system, an SIL-20A HT autosampler, an LC-20AT tandem pump system, a DGU-20A degasser unit, a CTO20AC column oven, an RDI-10A refractive index detector, and $4 \times$ Waters Styragel columns (HT2, HT3, HT4, and HT5, each $300 \mathrm{~mm} \times 7.8 \mathrm{~mm}^{2}$, providing an effective molar mass range of 100-4 $\times 10^{6}$ ). $N, N$-Dimethylacetamide (DMAc) (containing $4.34 \mathrm{~g} \mathrm{~L}^{-1}$ lithium bromide (LiBr)) was used as an eluent with a flow rate of $1 \mathrm{~mL} / \mathrm{min}$ at $80^{\circ} \mathrm{C}$. Number $\left(M_{\mathrm{n}}\right)$ and weight average $\left(M_{\mathrm{w}}\right)$ molar masses were evaluated using Shimadzu LC Solution software. The GPC columns 
were calibrated with low dispersity PMMA and PSt standards, and molar masses are reported as PMMA equivalents, except for PMMA- $b$-PSt where PSt calibration was used.
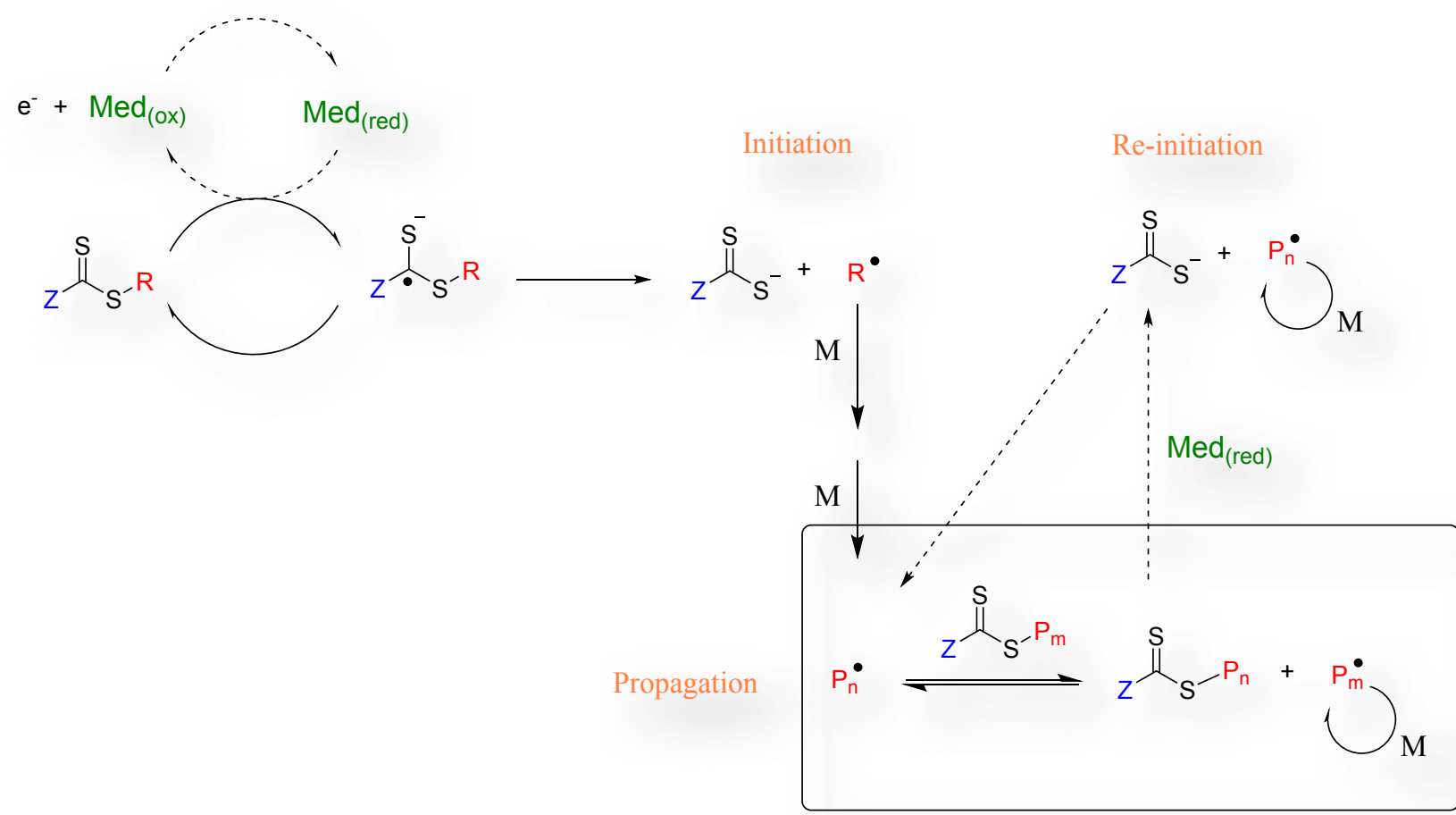

Scheme S1. Proposed scheme for mediated eRAFT polymerization
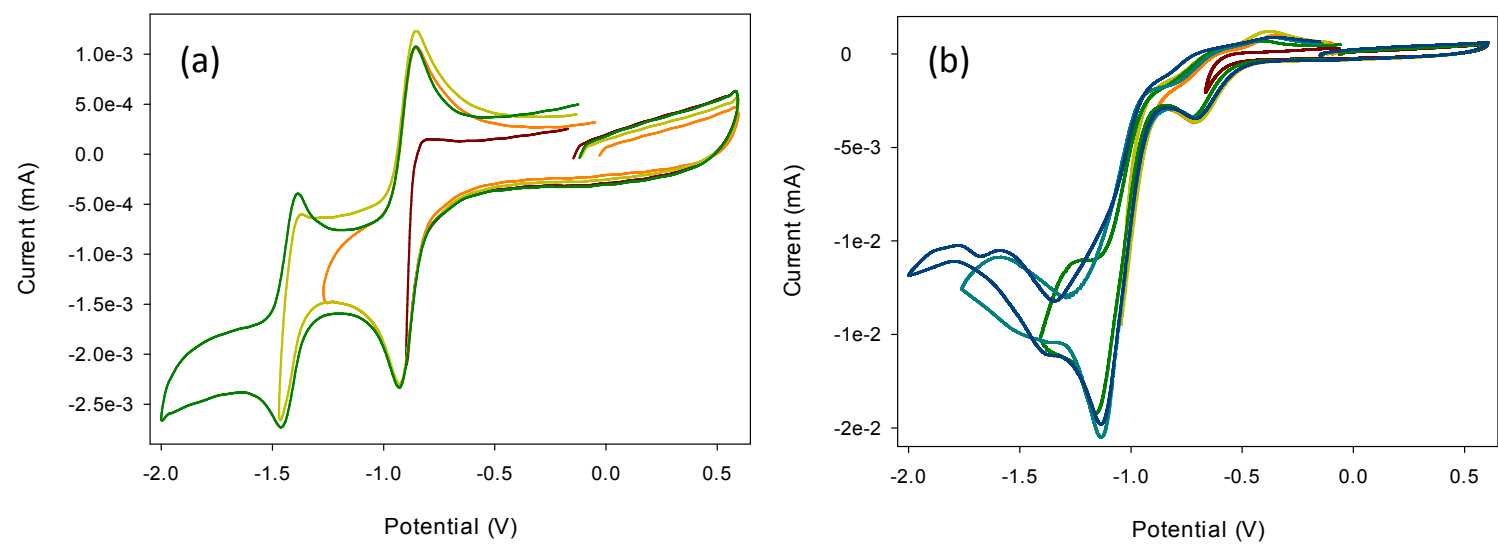

Figure S1. CVs of (a) AQ and (b) RAFT agent CDTPA in the presence of AQ, with CVs recorded to progressively more negative lower limits. CVs recorded in ACN with $0.1 \mathrm{M} \mathrm{TBAPF}_{6}$, W.E. $=1 \mathrm{~mm}$ GC disk (cleaned between each experiment), C.E. $=\mathrm{GC}$ rod, pseudoR.E. $=$ Ag wire. $v=100 \mathrm{mV} \mathrm{s}^{-1}$. 

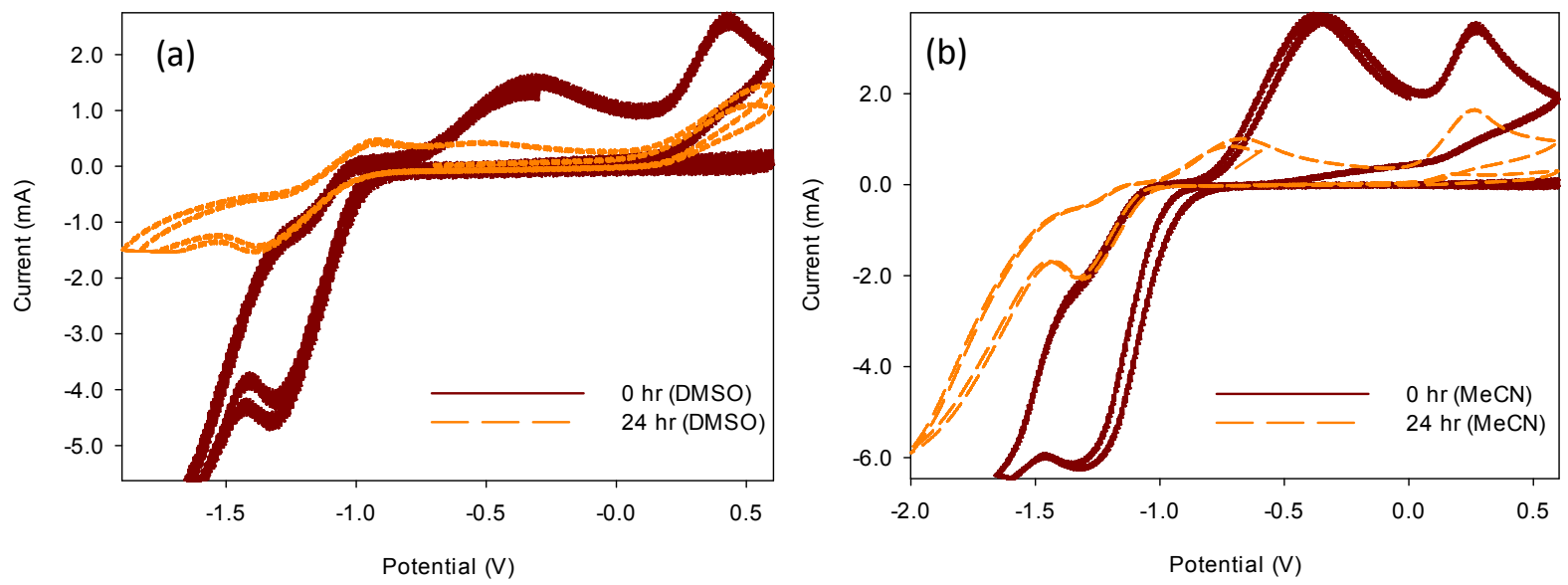

Figure S2. Pre-polymerization (red line) and post-polymerization (dashed orange line) CVs recorded in situ for mediated eRAFT of MMA in (a) DMSO and (b) ACN. W.E. $=$ GC rod, C.E. $=$ GC rod, R.E. $=\mathrm{Ag} / \mathrm{Ag}^{+} \cdot v=200 \mathrm{mV} \mathrm{s}^{-1}$.
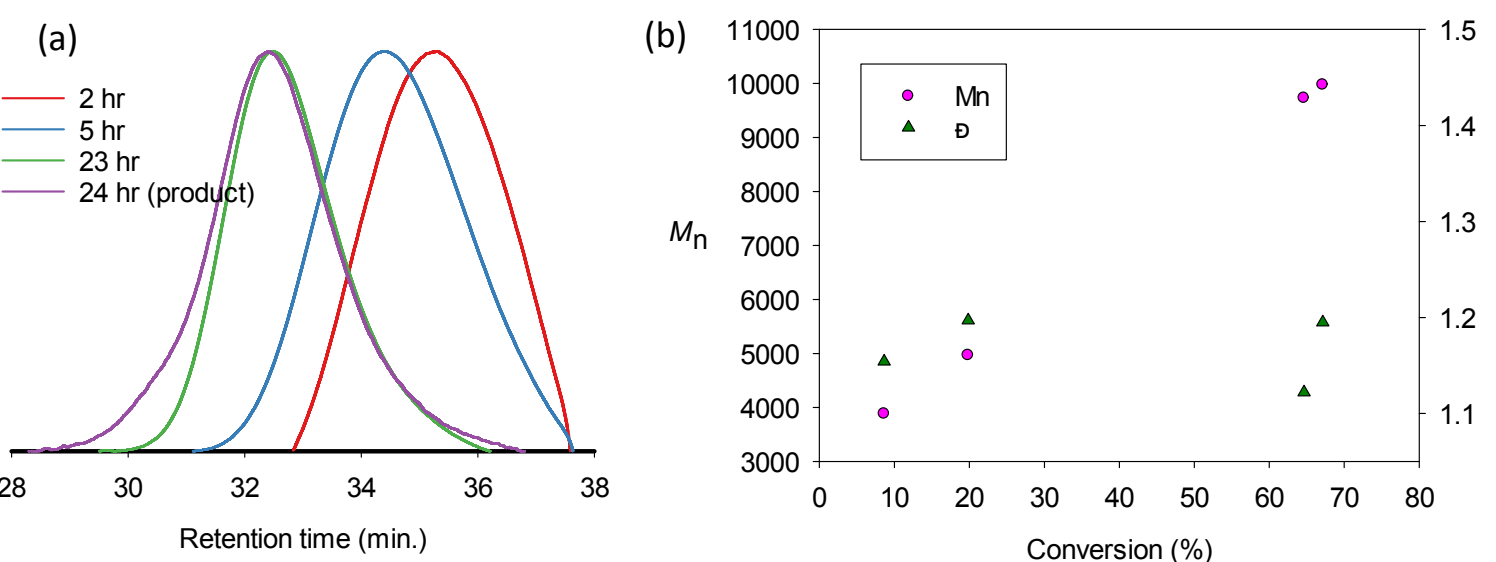

Figure S3. (a) Normalized GPC traces and (b) dispersity and $M_{\mathrm{n}}$ (determined by GPC, PMMA equiv.) as a function of conversion (determined by ${ }^{1} \mathrm{H}$ NMR spectroscopy) for mediated eRAFT polymerization of MMA (50 wt \% in DMSO, target $\mathrm{DP}=100,[\mathrm{MMA}]:[\mathrm{CDTPA}]:[\mathrm{AQ}]=100: 1: 0.1$, corresponding to Table 1 Entry 4). 
(a)

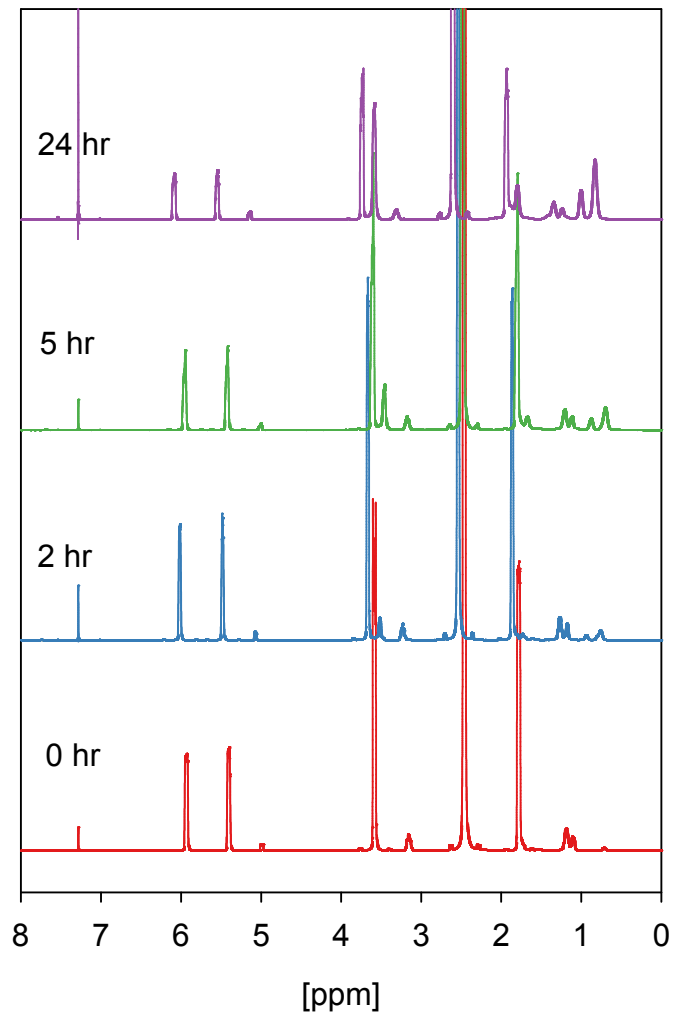

(b)

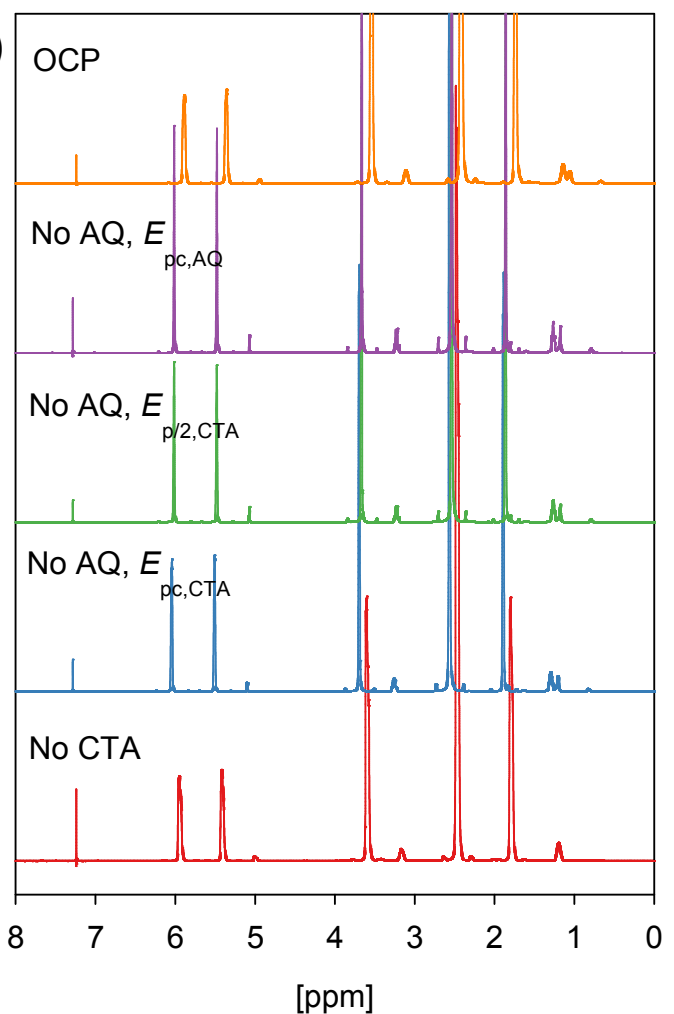

Figure S4. ${ }^{1} \mathrm{H}$ NMR spectra collected in $\mathrm{CDCl}_{3}$ with trioxane as internal standard $(\sim 5.0 \mathrm{ppm})$ for (a) mediated eRAFT polymerization of MMA (50 wt \% in DMSO, target DP $=100$, [MMA]: [CDTPA]: $[\mathrm{AQ}]=100: 1: 0.1$, corresponding to Table 1 Entry 4) at timepoints throughout electrolysis, and (b) mediated eRAFT controls without RAFT agent; without AQ at various applied potentials; and at open circuit potential (OCP) after $24 \mathrm{hr}$ electrolysis (corresponding to Table 1 Entries 1 H18).

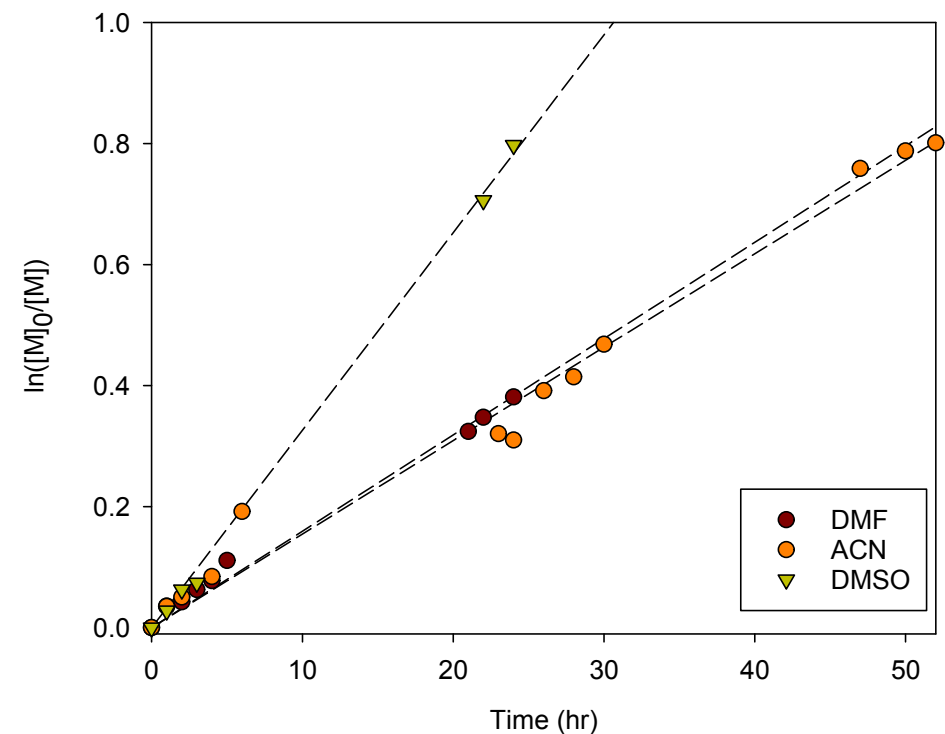

Figure S5. Monomer conversion for AQ-mediated eRAFT of MMA (50 wt \%) in various solvents (DMF, ACN, DMSO) as determined by ${ }^{1} \mathrm{H}$ NMR spectroscopy. [MMA]:[CDTPA]:[AQ] = 100:1:0.2. 

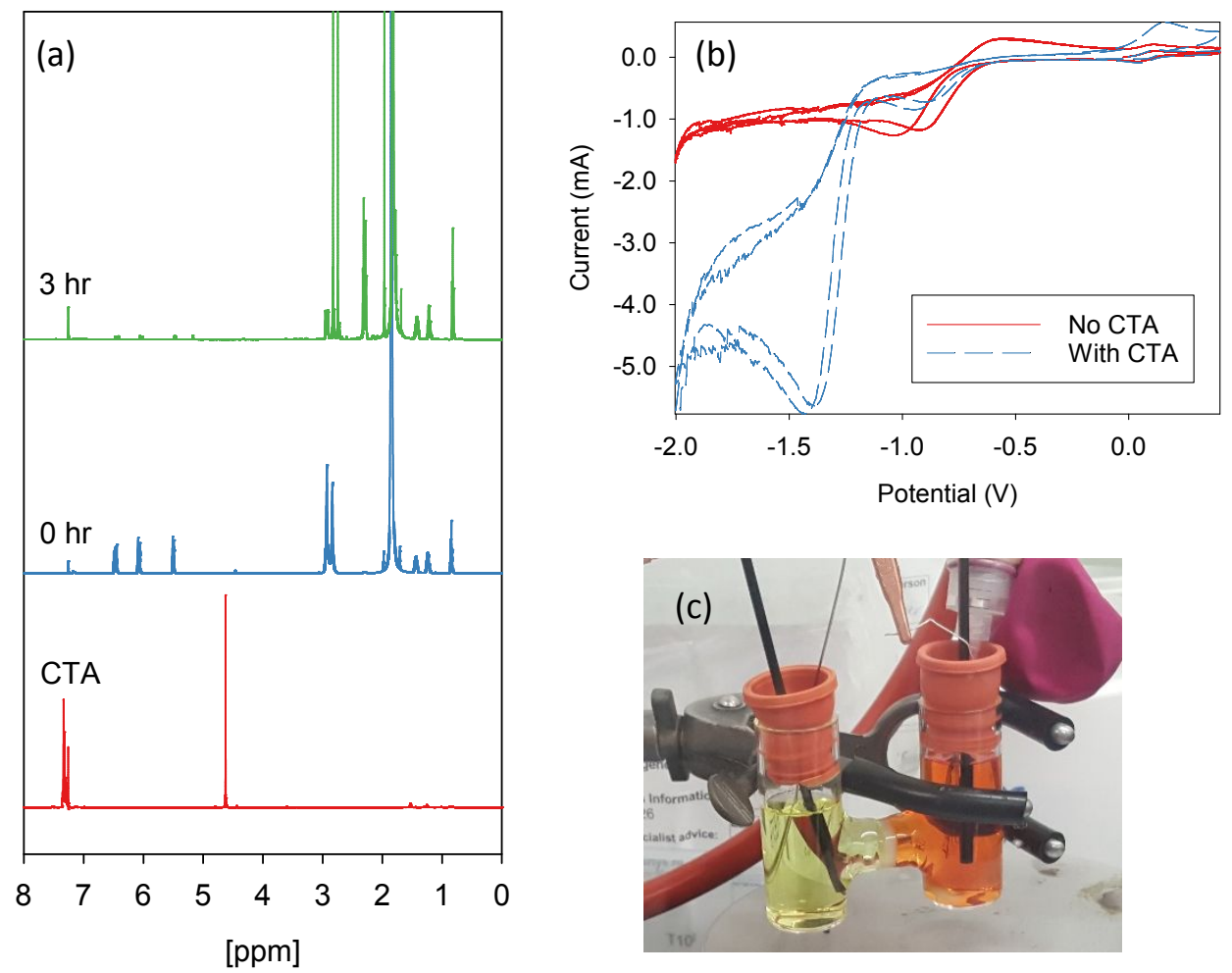

Figure S6. Electrolysis of RAFT agent (S,S-dibenzyl trithiocarbonate) in the presence of DMA monomer and 0.1 M $\mathrm{TBAPF}_{6}$ supporting electrolyte in ACN with small amounts of oxygen present in an H-cell without optimized degassing. (a) ${ }^{1} \mathrm{H}$ NMR spectra collected in $\mathrm{CDCl}_{3}$ of RAFT agent and electrolysis solution before and after electrolysis (NMR showed around $95 \%$ monomer conversion in $3 \mathrm{hr}$ but no polymer), (b) CVs recorded prior to electrolysis before (red line) and after (dashed blue line) adding RAFT agent to the nitrogen-purged cell, showing small amounts of oxygen in the CV even with degassing (W.E. = GC rod, C.E. = GC rod, pseudoR.E. = Pt wire, $v=100 \mathrm{mV} \mathrm{s}^{-1}$ ), and (c) photograph of the H-cell during electrolysis showing color change in the working electrode compartment attributed to degradation of the RAFT agent. 

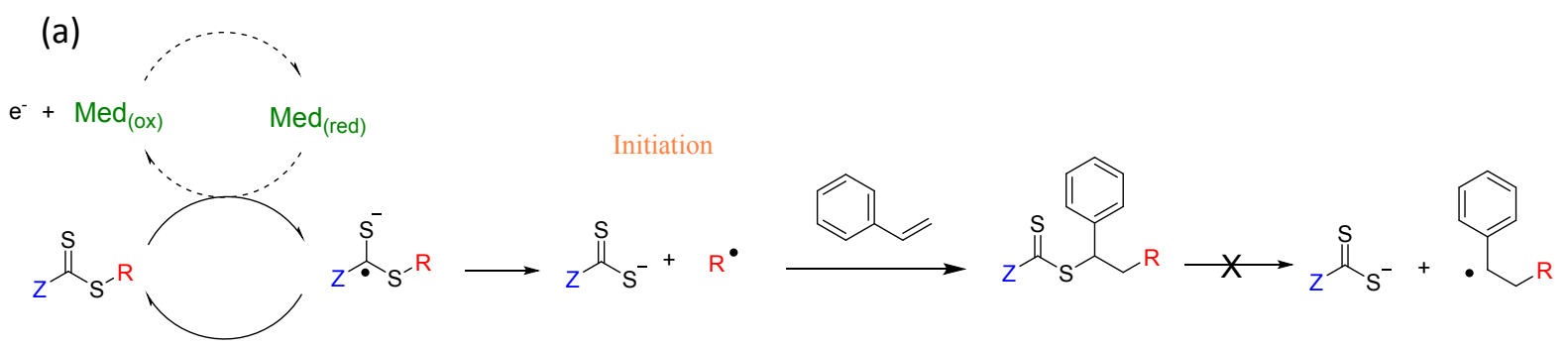

(b)

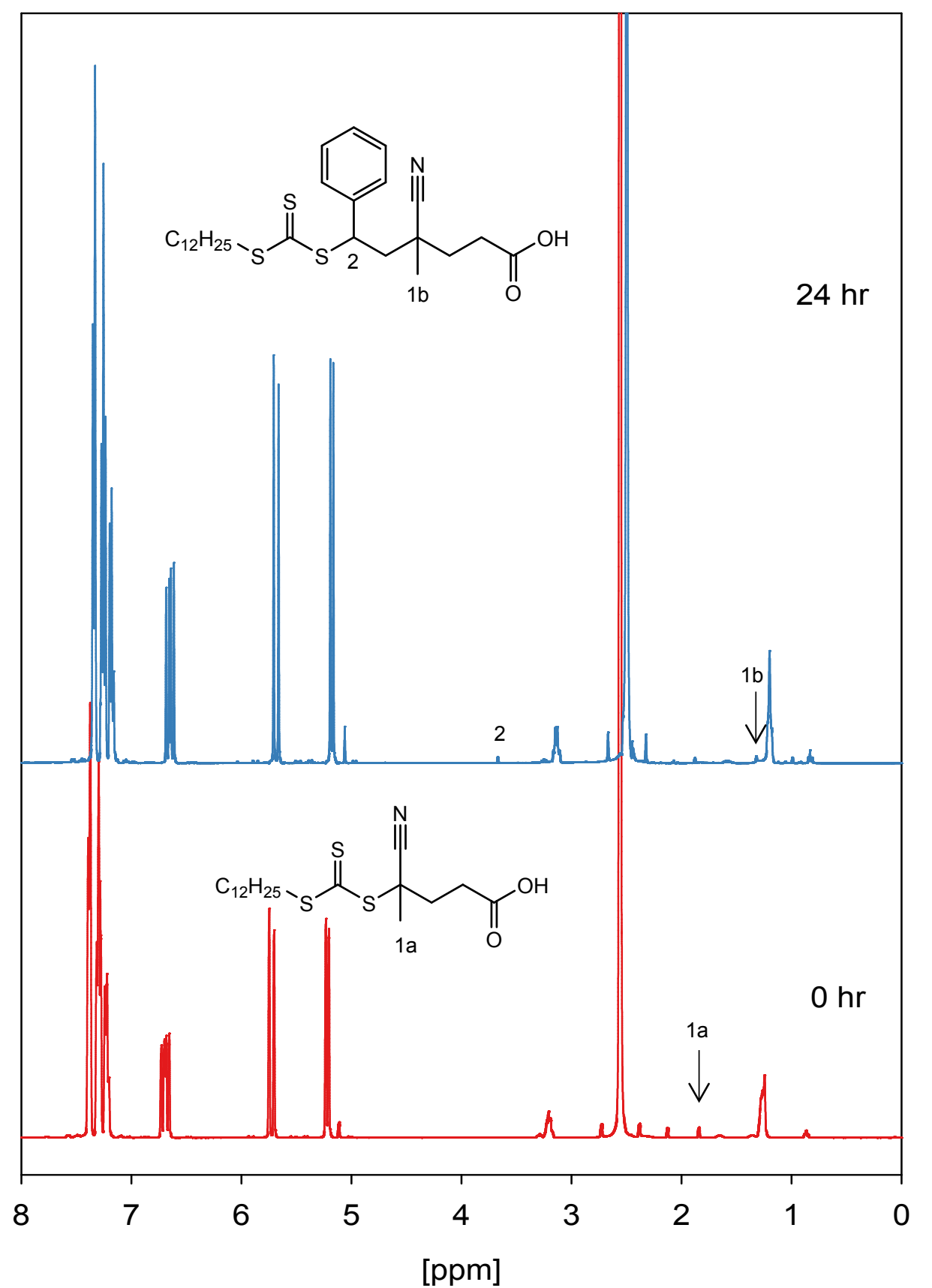

Figure S7. (a) Proposed scheme for eRAFT SUMI mechanism, and (b) ${ }^{1} \mathrm{H}$ NMR spectra collected in $\mathrm{CDCl}_{3}$ with trioxane as internal standard $(\sim 5.0 \mathrm{ppm})$ for mediated eRAFT of styrene (50 wt \% in DMSO, target DP $=100$, $[\mathrm{St}]:[\mathrm{CDTPA}]:[\mathrm{AQ}]=100: 1: 0.1$, corresponding to Table 1 Entry 20) showing peaks attributable to SUMI product after $24 \mathrm{hr}$ electrolysis. 

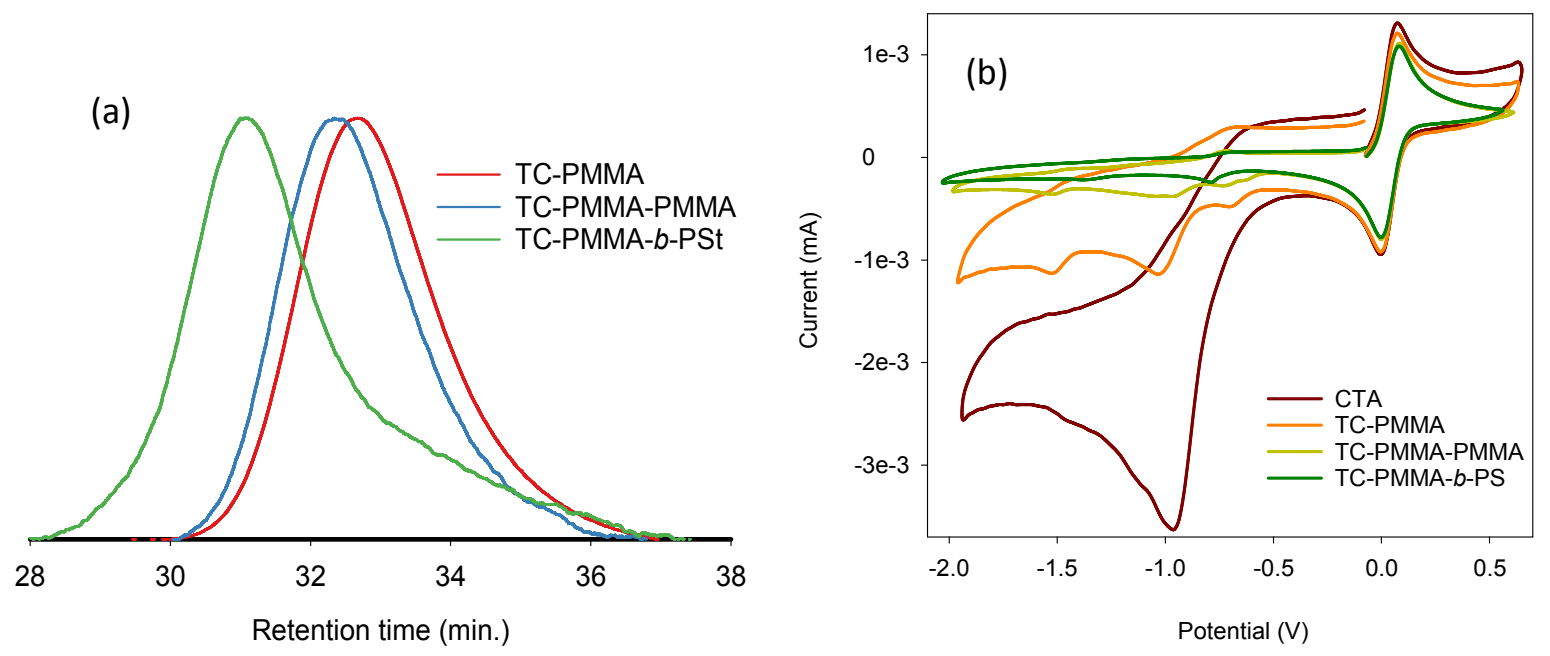

Figure S8. (a) Normalized GPC traces of mediated eRAFT product TC-PMMA, eRAFT chain extension product TCPMMA-PMMA, and RAFT block copolymerization TC-PMMA- $b$-PSt product; and (b) CVs of the same, and CDTPA RAFT agent, CVs recorded in ACN with $\leq 0.1 \mathrm{M} \mathrm{TBAPF}_{6}$, W.E. $=1 \mathrm{~mm} \mathrm{GC} \mathrm{disk,} \mathrm{C.E.}=$ GC rod, pseudoR.E. $=$ Ag wire. $v=100$ $\mathrm{mV} \mathrm{s}^{-1}$. CVs normalized to internal standard (decamethylferrocene) peak currents (concentrations for standard and for (macro)RAFT agents are not consistent across CVs). 